\title{
Health Outcomes Associated with Initiation of Basal Insulin After 1, 2, or $\geq 3$ Oral Antidiabetes Drug(s) Among Managed Care Patients with Type 2 Diabetes
}

\author{
Philip A. Levin, MD; Steve Zhou, PhD; Jasvinder Gill, PhD; and Wenhui Wei, PhD, MS, MBA
}

\begin{abstract}
BACKGROUND: Type 2 diabetes mellitus (T2DM) is a progressive disease. Despite starting with single oral antidiabetes drug (OAD) therapy and then adding $\mathrm{OAD}(\mathrm{s})$, most patients eventually require insulin therapy to achieve and maintain glycemic control. The timely initiation of insulin therapy could help patients with T2DM whose glycemic control is not adequately maintained using OADs alone.
\end{abstract}

OBJECTIVE: To describe and compare baseline characteristics and assess real-world health outcomes associated with initiating basal insulin after 1 OAD, 2 OADs, or $\geq 3$ OADs among T2DM patients.

METHODS: Data were analyzed from adult T2DM patients in a U.S. managed care claims database (IMPACT) who initiated a basal insulin (from January 1,2001 , to December 31,2011 ) with continuous health plan enrollment for 6 months before (baseline) and 12 months after (follow-up) insulin initiation and who had at least $1 \mathrm{OAD}$ prescription. Outcome measures according to the number of OADs used were (a) treatment discontinuation, (b) glycated hemoglobin (A1c) levels, (c) proportion of patients experiencing hypoglycemia, (d) health care resource utilization, and (e) costs.

RESULTS: Data from 71,988 patients were included (1 OAD: 19,168 patients [26.6\%]; 2 OADs: 29,112 [40.4\%]; and $\geq 3$ OADs: 23,708 [32.9\%]). All baseline characteristics, except nephropathy, were significantly different across the 3 groups. At baseline, when compared with the 1 OAD or 2 OADs groups, the $\geq 3$ OADs group was less likely to be female or have macrovascular disease and had experienced fewer hypoglycemic events and hospitalization as well as lower costs. At follow-up, treatment discontinuation rates were $36.0 \%, 27.6 \%$, and $21.4 \%$ for the $10 \mathrm{AD}, 20 \mathrm{ADs}$, and $\geq 3$ OADs groups, respectively. A1c reduction was $-1.33 \%,-1.05 \%$, and $-0.86 \%$, respectively. The proportion of patients experiencing any hypoglycemia was $4.7 \%, 3.8 \%$, and $3.3 \%$ at baseline; and $3.7 \%, 3.5 \%$, and $3.1 \%$ at follow-up for the 1 OAD, 2 OADs, and $\geq 3$ OADs groups, respectively. In all 3 groups, health care costs decreased compared with baseline, particularly in the $10 A D$ and 2 OADs groups, with decreased inpatient costs offsetting increased drug costs.

CONCLUSIONS: This real-world analysis shows that there are significant baseline differences in patients with T2DM on 1 OAD, 2 OADs, or $\geq 3$ OADs when adding insulin therapy. All 3 groups had significant improvements in clinical and economic outcomes compared with baseline, yet at different magnitudes. These data contribute to a growing body of evidence supporting the timely initiation of insulin therapy for T2DM patients not maintaining glycemic control with OADs.

J Manag Care Spec Pharm. 2015;21(12):1172-81

Copyright $\odot 2015$, Academy of Managed Care Pharmacy. All rights reserved.

\section{What is already known about this subject}

Type 2 diabetes mellitus (T2DM) is a progressive disease and so many patients eventually require insulin to achieve and maintain good glycemic control.

However, patients often delay insulin initiation, even after years of inadequate glycemic control on $\geq 2$ oral antidiabetes drugs (OADs).

The benefits of early insulin therapy initiation have been demonstrated in clinical trials.

\section{What this study adds}

This study provides real-world outcomes regarding clinical and economic benefits of insulin initiation in patients with T2DM uncontrolled with 1 OAD, 2 OADs, or $\geq 3$ OADs.

Patients with T2DM who were previously treated with 1 OAD, 2 OADs, or $\geq 3$ OADs and who initiated basal insulin therapy experienced significant benefits in clinical outcomes, including improvements in glycated hemoglobin and decreased risk of hypoglycemia, as well as in economic outcomes, including lower health care resource utilization and lower health care costs.

This real-world, observational, descriptive study contributes to a growing body of evidence suggesting that early initiation of insulin therapy for patients with T2DM who are unable to maintain adequate glycemic control with OADs alone can benefit from treatment intensification early in the disease course.

$\mathrm{B}$ ecause of the progressive nature of type 2 diabetes mellitus (T2DM), ${ }^{1,2}$ a majority of patients eventually require treatment with insulin in order to achieve and maintain acceptable glycemic control. ${ }^{3}$ A growing body of evidence suggests that timely initiation of insulin therapy is clinically beneficial. Ten-year follow-up data from the United Kingdom Prospective Diabetes study indicates that early treatment with insulin reduces the risk of macrovascular disease in patients with $\mathrm{T} 2 \mathrm{DM},{ }^{4}$ in addition to insulin's previously reported benefits on decreasing the risk of microvascular complications. ${ }^{5,6}$ Early insulin therapy may also restore and maintain betacell function to a greater extent than oral antidiabetes drugs (OADs) alone. ${ }^{7}$ 
Treatment guidelines from the American Diabetes Association (ADA) published before 2012 did not include guidelines about the benefits of timely initiation of insulin therapy. ${ }^{8}$ This lack of guidance may have contributed to a delay in insulin initiation until patients are no longer achieving adequate glycemic control..$^{9-11}$ This practice (i.e., delaying insulin initiation) has contributed to many patients having elevated long-term glycated hemoglobin (Alc) levels, with estimates indicating that Alc levels may be over the $7.0 \%$ target for as long as 10 years by the time insulin therapy is initiated. ${ }^{10}$ In addition, half of patients with T2DM delay the initiation of insulin therapy for about 5 years after the failure of OADs. ${ }^{11}$

Treatment guidelines from the ADA published since 2012 recommend the immediate initiation of insulin in newly diagnosed T2DM patients who have pronounced symptoms or elevated glucose or Alc levels. ${ }^{12-14}$ This recommendation is based on expert consensus or clinical experience. Insulin is also 1 of the 3 options - along with a second oral agent and a glucagon-like peptide-1 (GLP-1) receptor agonist-recommended as a second-line therapy in patients with inadequate glycemic control despite monotherapy with a noninsulin agent such as metformin..$^{12-14}$

The guidelines from the American Association of Clinical Endocrinologists recommend initiating insulin in patients who have Alc $>8.0 \%$; in those who are already being treated with at least 2 OADs or GLP-1 receptor agonist therapy; or in patients who are unlikely to achieve their Alc targets on further OADs. ${ }^{15}$ The clinical and economic benefits of early initiation of insulin therapy, however, remain to be determined, as realworld clinical practice data associated with the timely initiation of basal insulin is limited.

Several factors act as barriers to the timely initiation of insulin therapy. These include patient reluctance to start using insulin because of concerns over possible weight gain and hypoglycemic events, fear of injections, and a sense of personal failure. ${ }^{16-18}$ Physicians might share patient concerns about the adverse effects of insulin, and they also could be apprehensive about the resources that are required to educate patients and manage insulin use..$^{18,19}$

Although studies suggest that pharmacy-related costs can increase with the initiation of insulin after the failure of OADs alone, other studies have shown no significant differences in overall claims and diabetes-related health care utilization claims and costs. ${ }^{20,21}$ However, the economic benefits of early intervention with insulin have not been thoroughly investigated. ${ }^{22}$

To further assess the clinical benefits of early initiation of insulin therapy and provide data regarding possible economic benefits, we investigated real-world outcomes associated with the initiation of basal insulin therapy in patients with T2DM who were previously treated with 1 OAD, 2 OADs, or $\geq 3$ OADs in a managed care setting. The objective of this study was to investigate the patient characteristics when initiating insulin at different stages of the disease by using the number of baseline OADs as a proxy for diabetes disease severity, and to describe the outcomes for each patient group before and after insulin initiation.

\section{Methods}

This was a retrospective, observational study of data from a U.S. national managed care database (IMPACT) that comprises about 50 U.S. health care plans and contains medical and pharmacy claims, eligibility data, and laboratory results for 107 million patients, of whom 73\% had pharmacy benefits and $18 \%$ had laboratory results.

Adult patients (aged $\geq 18$ years) with T2DM who initiated a basal insulin from January 1, 2001, to December 31, 2011, and who had continuous health plan enrollment for 6 months before (baseline period) and 12 months after (followup period) initiation of basal insulin therapy were eligible for inclusion. Patients must have received at least 1 prescription for an OAD (metformin, sulfonylureas, thiazolidinediones, dipeptidyl peptidase [DPP]-4 inhibitors, meglitinides, alphaglucosidase inhibitors) in the baseline period for their data to be included in the analysis. Data from patients using injectable antidiabetic medications (pramlintide, GLP-1 receptor agonists) or insulin during the baseline period were excluded from the analysis.

Because of a lack of diabetes duration in the claim data, we used the number of OADs before insulin initiation as a proxy for disease progression. This assumption was based on previous and current diabetes guidelines on pharmacological therapy for hyperglycemia in T2DM patients that recommend OAD monotherapy (metformin or sulfonylureas) first, and then initiation of insulin by adding it to either 1 OAD or subsequent combinations of OADs. ${ }^{23,24}$ Therefore, for the current analyses, patients were stratified according to whether they used 1 OAD, 2 OADs, or $\geq 3$ OADs during the baseline period.

\section{Study Outcomes}

Baseline patient demographic and disease characteristics were reported, including the proportions of patients with different types of comorbidities, as well as an evaluation of the overall health of the patient (measured by the Charlson Comorbidity Index [CCI]). In addition, antidiabetes treatment and medication use among these patients was reported.

One-year follow-up outcomes were insulin treatment discontinuation, Alc levels, proportion of patients experiencing hypoglycemia, health care resource utilization, and health care costs.

Study medication was considered discontinued if the prescription was not refilled during the last quarter of the 1-year follow-up period..$^{25}$ Glycemic control was measured by Alc levels at the end of the 1-year follow-up period and by change from baseline Alc. 
TABLE 1 Demographics, Clinical Characteristics, and Comorbidities During the Baseline Period

\begin{tabular}{|c|c|c|c|c|c|c|}
\hline \multirow{2}{*}{$\begin{array}{l}\text { Characteristic } \\
\text { Age, years, mean (SD) }\end{array}$} & \multicolumn{2}{|c|}{1 OAD $(\mathrm{N}=19,168)$} & \multicolumn{2}{|c|}{2 OADs $(\mathrm{N}=29,112)$} & \multicolumn{2}{|c|}{$\geq 3$ OADs $(\mathrm{N}=23,708)$} \\
\hline & 54.2 & $(13.00)$ & 55.3 & $(11.20)$ & 55.7 & $(10.00)$ \\
\hline Female & 9,507 & $(49.6)$ & 13,291 & $(45.7)$ & 8,905 & $(37.6)$ \\
\hline Alc, \%, mean (SD) & 9.33 & $(2.53)$ & 9.50 & $(2.16)$ & 9.31 & $(1.92)$ \\
\hline Charlson Comorbidity Index, mean (SD) & 1.00 & $(1.72)$ & 0.80 & $(1.51)$ & 0.61 & $(1.29)$ \\
\hline Any hypoglycemia event & 904 & $(4.7)$ & 1,128 & $(3.9)$ & 797 & $(3.4)$ \\
\hline Myocardial infarction & 802 & $(4.2)$ & 1,150 & $(4.0)$ & 691 & $(2.9)$ \\
\hline Congestive heart failure & 2,237 & $(11.7)$ & 2,760 & $(9.5)$ & 1,657 & $(7.0)$ \\
\hline Peripheral vascular disease & 1,285 & $(6.7)$ & 1,849 & $(6.4)$ & 1,249 & $(5.3)$ \\
\hline Cerebrovascular disease & 1,350 & $(7.0)$ & 1,871 & $(6.4)$ & 1,164 & $(4.9)$ \\
\hline Hypertension & 11,460 & (59.8) & 18,499 & $(63.5)$ & 15,557 & $(65.6)$ \\
\hline Hyperlipidemia & 9,441 & (49.3) & 16,860 & $(57.9)$ & 15,621 & $(65.9)$ \\
\hline Neuropathy & 2,012 & $(10.5)$ & 3,271 & $(11.2)$ & 2,673 & (11.3) \\
\hline Nephropathy & 933 & (4.9) & 1,463 & $(5.0)$ & 1,153 & $(4.9)$ \\
\hline Retinopathy & 1,537 & $(8.0)$ & 2,787 & $(9.6)$ & 2,735 & $(11.5)$ \\
\hline Obesity & 2,085 & (10.9) & 2,943 & $(10.1)$ & 2,240 & $(9.4)$ \\
\hline
\end{tabular}

Note: Values are $n(\%)$ unless otherwise stated.

All characteristics were significantly different across groups ( $P<0.017$ for neuropathy; $P<0.001$ for all others), except nephropathy $(P=0.622)$.

$A l c=$ glycated hemoglobin; $O A D=$ oral antidiabetes drug; $S D=$ standard deviation.

Hypoglycemic events were defined as a health care encounter (outpatient, inpatient, or emergency department [ED] visit) with a primary or secondary International Classification of Diseases, Ninth Revision, Clinical Modification (ICD-9-CM) diagnosis code for hypoglycemia (ICD-9-CM codes: 250.8-diabetes with other specified manifestations; 251.0-hypoglycemic coma; 251.1—other specified hypoglycemia; or 251.2-hypoglycemia, unspecified). ${ }^{26}$ Hypoglycemia was assessed as the prevalence of hypoglycemia-related events, defined as the percentage of patients with at least 1 hypoglycemia-related event during follow-up.

Health care resource utilization was measured as the number of outpatient visits, ED visits, inpatient admissions, endocrinologist visits, and inpatient length of stay (days). Diabetesrelated health care resource utilization included claims with a primary or secondary diagnosis of diabetes (ICD-9-CM code: 250.xx)

A cost analysis was performed on health care costs during baseline, and the first and second 6 months of follow-up. Costs were considered diabetes-related if the claim had a primary or secondary diagnosis ICD-9-CM code of 250.xx, or if the claim was a pharmacy claim for antidiabetes medication and supplies. Total costs were calculated as aggregates of inpatient, outpatient, and ED visits, and prescription costs.

For hypoglycemia and health care resource utilization and costs, an evaluation was also made at 6 months after initiation of basal insulin therapy.

\section{Statistical Analysis}

Descriptive statistical analyses were conducted to assess treatment patterns and study outcomes. To assess baseline dif- ferences between patients who were on different numbers of OADs, analysis of variance was used for continuous variables, and $\mathrm{X}^{2}$ test was used for categorical variables. Within each group of patients ( 1 OAD, 2 OADs, and $\geq 3$ OADs), McNemar's test was used to assess differences between baseline and followup for the proportion of patients experiencing hypoglycemia, and paired t-test was used for health care costs. Health care costs were highly skewed; therefore, sensitivity analyses with nonparametric tests were conducted using Kruskal-Wallis test to examine differences in median/distribution of baseline costs between the 3 groups, and Wilcoxon signed-rank test to compare baseline and follow-up health care costs within each group.

Because of the descriptive nature of the study and the significant baseline differences, no statistical tests were conducted on comparisons of follow-up outcomes between the 3 groups. Statistical analyses were conducted using SAS software package 9.3 (SAS Institute, Cary, NC).

\section{Results}

\section{Baseline Characteristics}

Data from a total of 71,988 patients were included in this analysis. During the baseline period, 19,168 (26.6\%) patients were treated with 1 OAD, 29,112 (40.4\%) patients with 2 OADs, and 23,708 (32.9\%) patients with $\geq 3$ OADs. There were significant differences in baseline demographic and disease characteristics among the 3 groups, except nephropathy (Table 1). When compared with patients in the 1 OAD or 2 OADs groups, patients in the $\geq 3$ OADs group were slightly older, less likely to be female, healthier (as measured by the CCI), and with a lower Alc level and lower risk of hypoglycemia, macrovascular 


\begin{tabular}{|c|c|c|c|}
\hline & $\begin{array}{c}1 \text { OAD } \\
(\mathrm{N}=19,168)\end{array}$ & $\begin{array}{c}2 \text { OADs } \\
(\mathrm{N}=29,112)\end{array}$ & $\begin{array}{c}\geq 3 \text { OADs } \\
(\mathrm{N}=23,708)\end{array}$ \\
\hline \multicolumn{4}{|l|}{ Baseline OAD, n (\%) } \\
\hline Metformin & $9,148(47.7)$ & $23,781 \quad(81.7)$ & $22,856(96.4)$ \\
\hline Sulfonylurea & $6,703(35.0)$ & $21,752(74.7)$ & $21,948(92.6)$ \\
\hline Thiazolidinedione & $2,177(11.4)$ & $8,715(29.9)$ & $19,503(82.3)$ \\
\hline DPP-4 inhibitor & $633 \quad(3.3)$ & $2,852 \quad(9.8)$ & $6,542(27.6)$ \\
\hline Meglitinide & $460 \quad(2.4)$ & $955 \quad(3.3)$ & $2,449(10.3)$ \\
\hline Alpha-glucosidase inhibitor & $47 \quad(0.2)$ & $169 \quad(0.6)$ & $812 \quad(3.4)$ \\
\hline Metformin + sulfonylurea & NA & $16,962(58.3)$ & NA \\
\hline $\begin{array}{l}\text { Metformin }+ \\
\text { thiazolidinedione }\end{array}$ & NA & $4,650(16.0)$ & NA \\
\hline $\begin{array}{l}\text { Metformin + sulfonylurea }+ \\
\text { thiazolidinedione }\end{array}$ & NA & NA & $14,545(61.4)$ \\
\hline $\begin{array}{l}\text { Metformin+sulfonylurea }+ \\
\text { DPP-4 inhibitor }\end{array}$ & NA & NA & $3,160(13.3)$ \\
\hline \multicolumn{4}{|c|}{ Type of initiated basal insulin, $\mathrm{n}(\%)$} \\
\hline Glargine & $13,201(68.9)$ & $21,644(74.3)$ & $18,112(76.4)$ \\
\hline Detemir & $2,274(11.9)$ & $3,889(13.4)$ & $3,429(14.5)$ \\
\hline Neutral protamine Hagedorn & $3,648(19.0)$ & $3,529(12.1)$ & $2,133 \quad(9.0)$ \\
\hline Lente/ultralente & $45 \quad(0.2)$ & $50 \quad(0.2)$ & $34 \quad(0.1)$ \\
\hline \multicolumn{4}{|c|}{$\begin{array}{l}\text { Note: The proportion of patients using each treatment was significantly different } \\
\text { across the groups }(P<0.001) \text {, with the exception of lente/ultralente insulin } \\
(P=0.078) \text {. } \\
\text { DPP-4 = dipeptidyl peptidase- } 4 ; N A=\text { not applicable; } O A D=\text { oral antidiabetes drug. }\end{array}$} \\
\hline
\end{tabular}

disease (myocardial infarction, congestive heart failure, peripheral vascular disease, and cerebrovascular disease), and obesity. However, these patients had higher rates of retinopathy, hypertension, and hyperlipidemia.

Almost all of the patients in the $\geq 3$ OADs group were previously treated with metformin, with $>80 \%$ of patients having received sulfonylureas and thiazolidinedione. The most prevalent 3 OAD combinations were metformin + sulfonylurea + thiazolidinedione (61.4\%), followed by metformin + sulfonylurea+DPP-4 inhibitor (13.3\%). For the 2 OADs group, the most prevalent 2 OAD combinations were metformin + sulfonylurea (58.3\%), followed by metformin + thiazolidinedione (16.0\%) (Table 2).

With regard to the basal insulin initiated at the end of the baseline period, insulin glargine was the most commonly prescribed basal insulin in each of the 3 groups (insulin glargine $68.9 \%-76.4 \%$, insulin detemir $11.9 \%-14.5 \%$, neutral protamine Hagedorn (NPH) 9.0\%-19.0\%, and insulin lente/ultralente $<1 \%$ ). However, a significantly greater proportion of patients in the 2 OADs and $\geq 3$ OADs groups used insulin glargine or insulin detemir compared with those in the 1 OAD group (glargine: $74.3 \%$ and $76.4 \%$ vs. $68.9 \%$, respectively; $P<0.001$; detemir: $13.4 \%$ and $14.5 \%$ vs. $11.9 \%$, respectively; $P<0.001$ ). In contrast, a significantly greater proportion of patients in the 1 OAD group initiated therapy with NPH $(19.0 \%$ vs. $12.1 \%$ in
TABLE 3 All-Cause Health Care Resource Utilization $^{\mathrm{a}}$

\begin{tabular}{|c|c|c|c|}
\hline Resource, n (\%) & $\begin{array}{c}1 \text { OAD } \\
(\mathrm{N}=19,168)\end{array}$ & $\begin{array}{c}2 \text { OADs } \\
(\mathrm{N}=29,112)\end{array}$ & $\begin{array}{c}\geq 3 \text { OADs } \\
(\mathrm{N}=23,708)\end{array}$ \\
\hline \multicolumn{4}{|l|}{ Hospitalization } \\
\hline Baseline & $5,321(27.8)$ & $6,131(21.1)$ & $3,546(15.0)$ \\
\hline First 6 months follow-up & $5,145(26.8)$ & $5,602(19.2)$ & $3,251(13.7)$ \\
\hline Second 6 months follow-up & $3,036(15.8)$ & $3,569(12.3)$ & $2,206(9.3)$ \\
\hline \multicolumn{4}{|l|}{ Office visit } \\
\hline Baseline & $18,582(96.9)$ & $28,420(97.6)$ & $23,403(98.7)$ \\
\hline First 6 months follow-up & $18,728(97.7)$ & $28,528(98.0)$ & $23,268(98.1)$ \\
\hline Second 6 months follow-up & $18,094(94.4)$ & $27,715(95.2)$ & $22,773(96.1)$ \\
\hline \multicolumn{4}{|l|}{ ED visit } \\
\hline Baseline & $6,325(33.0)$ & $7,993(27.5)$ & $5,084(21.4)$ \\
\hline First 6 months follow-up & $5,293(27.6)$ & $7,002(24.1)$ & $4,606(19.4)$ \\
\hline Second 6 months follow-up & $4,391(22.9)$ & $6,038(20.7)$ & $4,091(17.3)$ \\
\hline \multicolumn{4}{|l|}{ Endocrinology visit } \\
\hline Baseline & $2,640(13.8)$ & $4,564(15.7)$ & $4,796(20.2)$ \\
\hline First 6 months follow-up & $4,153(21.7)$ & $6,328(21.7)$ & $5,950(25.1)$ \\
\hline Second 6 months follow-up & $3,438(17.9)$ & $5,685(19.5)$ & $5,498(23.2)$ \\
\hline
\end{tabular}

Notes: At baseline, the proportion of patients with each type of health care resource utilization was significantly different across the groups $(P<0.001)$.

During the first and second 6 months of follow-up, the proportions of patients with each type of health care resource utilization in all 3 groups were significantly different from baseline with a $P$ value of $<0.001$, except the proportion of patients in the 2 OADs group with office visits during the first 6 months $(P=0.001)$ and the proportion of patients in the 1 OAD group being hospitalized during the first 6 months $(P=0.005)$

${ }^{a}$ Number and proportion of patients requiring at least one hospital, office, ED, or endocrinology visit during each period.

$E D=$ emergency department $O A D=$ oral antidiabetes drug.

the 2 OAD group and $9.0 \%$ in the $\geq 3$ OADs group; $P<0.001$ ) (Table 2).

In terms of health care utilization, a significantly lower proportion of patients in the $\geq 3$ OADs group were hospitalized or had an ED visit for any cause during the baseline period compared with those in the 1 OAD or 2 OADs groups (hospitalization: $15.0 \%$ vs. $27.8 \%$ and $21.1 \%$, respectively; $P<0.001$; ED visit: $21.4 \%$ vs. $33.0 \%$ and $27.5 \%$, respectively; $P<0.001$ ) (Table 3). In contrast, a significantly greater proportion of patients in the $\geq 3$ OADs group had an office visit (98.7\%) or an endocrinology visit (20.2\%) during the baseline period compared with those in the 1 OAD or 2 OADs groups (office: $98.7 \%$ vs. $96.9 \%$ and 97.6\%; $P<0.001$; endocrinology: $20.2 \%$ vs. $13.8 \%$ and $15.7 \%$; $P<0.001)$.

Mean all-cause health care costs (in U.S. dollars) at baseline were significantly lower for patients in the $\geq 3$ OADs group compared with those in the $1 \mathrm{OAD}$ and 2 OADs groups ( $\$ 21,585$ vs. $\$ 32,880$ and $\$ 25,945$, respectively; $P<0.001$; Figure 1A). A similar pattern was observed for diabetes-related costs ( $\$ 9,531$ for 1 OAD, $\$ 8,604$ for 2 OADs, and $\$ 8,357$ for $\geq 3$ OADs; $P<0.001$ ) (Figure 1B). 


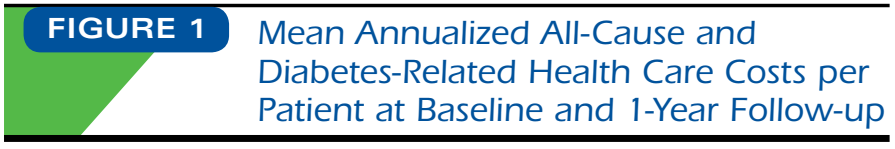

A. All-Cause Health Care Costs

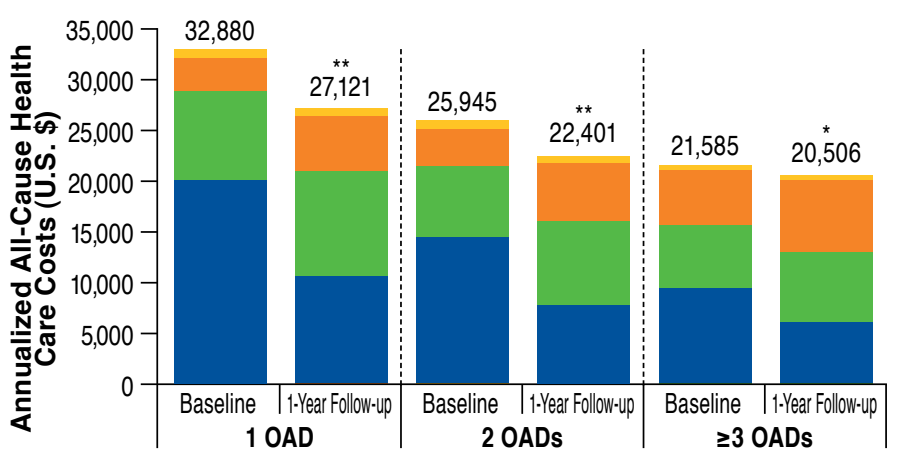

B. Diabetes-Related Health Care Costs

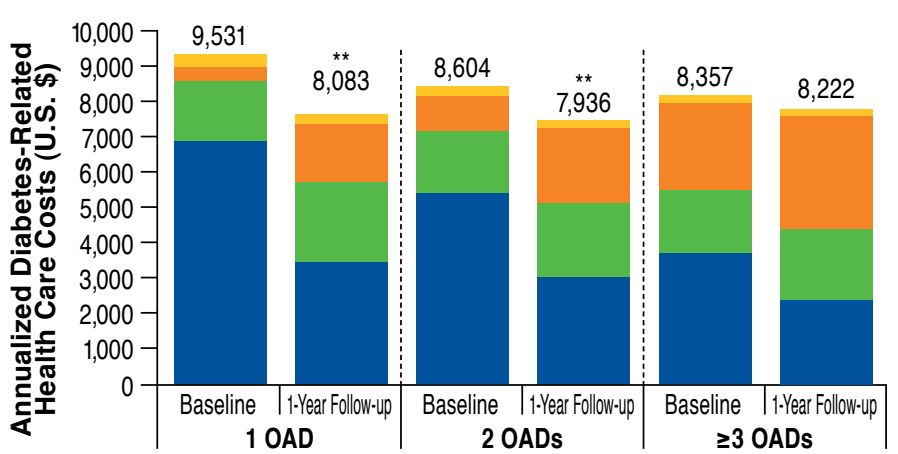

Inpatient Costs Outpatient Costs Drug Costs ED Costs

Notes: Numbers represent total health care costs per patient.

All differences in mean health care costs per patient at baseline were $P<0.001$ between groups, except for diabetes-related outpatient costs $(P=0.707)$.

During 1-year follow-up, mean total all-cause and diabetes-related health care costs in all 3 groups were significantly lower compared with baseline $\left({ }^{*} P=0.003\right.$ and $\left.{ }^{* *} P<0.001\right)$, except total diabetes-related health care costs in the $\geq 3$ OADs group $(P=0.285)$.

$E D=$ emergency department; $O A D=$ oral antidiabetes drug.

\section{One-Year Follow-up Outcomes}

Alc Reductions and Insulin Treatment Discontinuation. Among patients with follow-up Alc data available (1 OAD, $\mathrm{N}=3,293 ; 2$ OADs, $\mathrm{N}=5,397$; and $\geq 3$ OADs, $\mathrm{N}=4,945)$, mean ( \pm standard deviation) of Alc levels was significantly reduced from baseline by $-1.33 \%( \pm 2.49),-1.05 \%( \pm 2.11)$, and $-0.86 \%$ $( \pm 1.92)$ in the 1 OAD, 2 OADs, and $\geq 3$ OADs groups, respectively (all $P<0.001$ ) (Figure 2). By the end of the 1-year followup period, the discontinuation rates with insulin treatment were $36.0 \%, 27.6 \%$, and $21.4 \%$ for the 1 OAD, 2 OADs, and $\geq 3$ OADs groups, respectively. When the groups were stratified by discontinuation of insulin treatment, Alc reductions
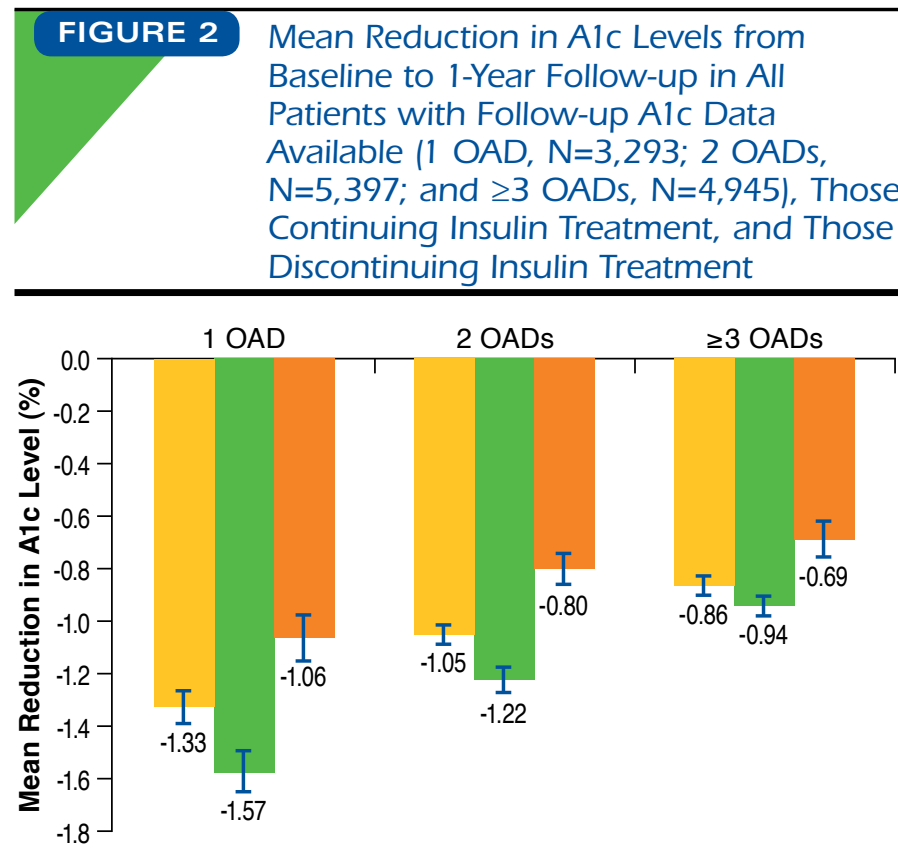

All Patients

Patients Continuing Insulin Treatment

Patients Discontinuing Insulin Treatment

Note: In the "All Patients" group, reductions in A1c level at 1-year follow-up were all significantly different from baseline in all groups $(P<0.001)$. Error bars represent standard errors.

Alc = glycated hemoglobin; OAD = oral antidiabetes drug.

were $-1.57 \%( \pm 2.43),-1.22 \%( \pm 2.12)$, and $-0.94 \%( \pm 1.87)$ for patients who did not discontinue insulin treatment by the end of the 1-year follow-up period; and $-1.06 \%( \pm 2.54),-0.80 \%$ $( \pm 2.06)$, and $-0.69 \%( \pm 2.02)$ for those who did discontinue treatment in the 1 OAD, 2 OADs, and $\geq 3$ OADs groups, respectively (Figure 2).

Hypoglycemia. In all 3 groups, the proportion of patients experiencing at least 1 hypoglycemic event increased slightly during the first 6 months of the follow-up period before decreasing during the second 6 months of the follow-up period compared with baseline (Figure 3). The proportions of patients who experienced any hypoglycemia were 4.7\%, 3.8\%, and $3.3 \%$ in the baseline period and decreased to 3.7\%, 3.5\%, and $3.1 \%$ during follow-up for the 1 OAD, 2 OADs, and $\geq 3$ OADs groups, respectively. The proportions of patients who experienced inpatient/ED-related hypoglycemia were low in the baseline period ( $1 \mathrm{OAD}, 3.0 \%$; 2 OADs, $2.3 \%$; and $\geq 3$ OADs, $1.6 \%$ ) and decreased during follow-up (1 OAD, 1.6\%; 2 OADs, $1.3 \%$; and $\geq 3$ OADs, $1.1 \%$ in the second 6 months of the follow-up period). 


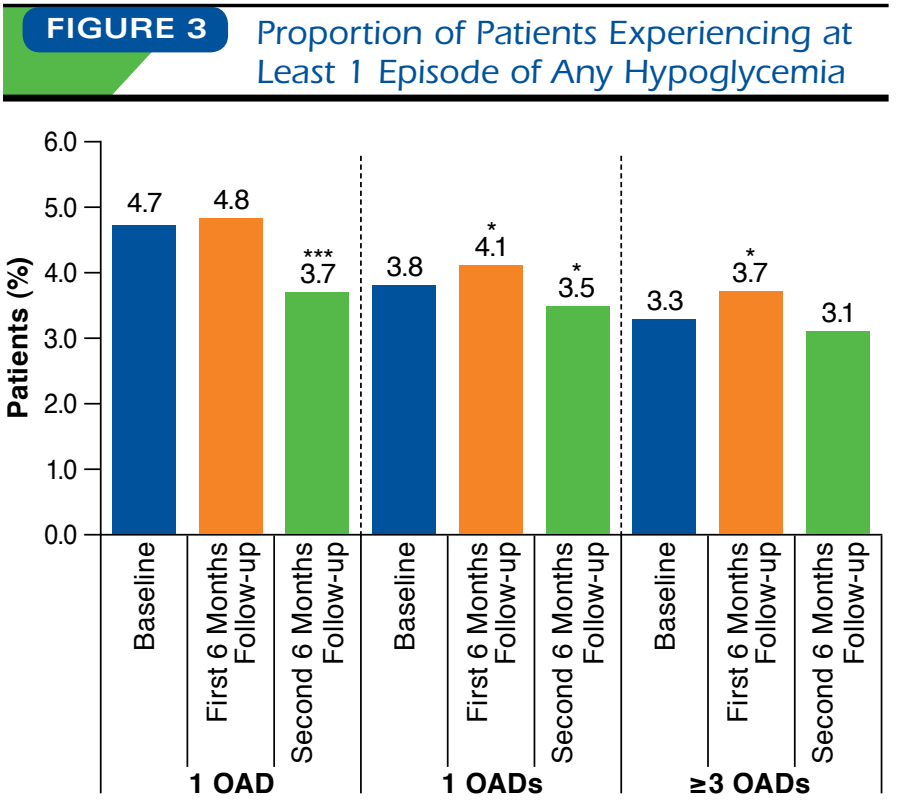

Note: Proportion of patients experiencing at least 1 episode of any hypoglycemia rates was significantly different between the 3 groups during the 6-month baseline period $(P<0.001)$. P values for differences between baseline and follow-up within each patient group were $* P<0.050,{ }^{*} P<0.010$, and $* * *<0.001$.

$O A D=$ oral antidiabetes drug.

Health Care Resource Utilization and Costs. The proportion of patients in each group with an endocrinology visit significantly increased during the follow-up period compared with baseline, whereas most other types of health care resource utilization significantly decreased following initiation of basal insulin therapy (Table 3).

All-cause and diabetes-related health care costs significantly decreased in the year following initiation of insulin therapy compared with baseline, except total diabetes-related health care costs in the $\geq 3$ OADs group (Figure 1B). The largest reduction in costs (in U.S. dollars) was observed in the 1 OAD group (all-cause costs were $-\$ 5,759$ and diabetes-related costs were $-\$ 1,448)$. This reduction was mainly due to decreased inpatient costs, which offset the increase in drug costs. Conversely, the smallest reduction in all-cause and diabetes-related health care costs occurred in the $\geq 3$ OADs group (all-cause costs were -\$1,079 and diabetes-related costs were $-\$ 135)$.

Nonparametric Wilcoxon signed-rank tests showed similar results with the exception of the $\geq 3$ OADs group, in which the annualized baseline and follow-up total diabetes-related health care costs were significantly different $(P<0.001)$. The data on median (range) all-cause and diabetes-related health care costs at baseline and follow-up are provided in Appendix (available in online article).

\section{Discussion}

This real-world study adds to the body of evidence supporting the initiation of basal insulin therapy in patients with T2DM who are not achieving glycemic control despite therapy with OADs. Additionally, it provides information on the economic advantages that could be realized with initiation of basal insulin therapy.

Significant differences at baseline among patients with T2DM who initiated basal insulin therapy in addition to treatment with 1 OAD, 2 OAD, or $\geq 3$ OADs were observed. In particular, these differences suggested that patients who initiated insulin following 1 OAD were sicker, in terms of the number of comorbidities, at baseline compared with those who initiated insulin therapy after 2 or $\geq 3$ OADs. These sicker patients had higher rates of macrovascular disease, hospitalizations, ED visits, and increased risk of hypoglycemia, as well as lower health care costs at baseline than patients in the other two groups in this analysis.

Although this finding seems counterintuitive, these results suggest that diabetes medical practice of insulin use during the time frame of this study (2001-2011) was focused on escalation to insulin treatment for sicker patients, possibly as a result of a medical encounter, after which insulin initiation was urged. Furthermore, these patients could have medical conditions limiting their options for additional OADs or a rapid worsening of Alc levels, urging initiation with insulin therapy.

According to the ADA guidelines, the choice of therapy should be based on consideration of efficacy, cost-adverse events profile, risk of weight change, presence of comorbidities, risk of hypoglycemia, age, and patient preferences. ${ }^{14}$ Our analysis did not include all these factors (e.g., adverse events profile, impact on body weight, and patient preferences), but did show notable differences in some baseline characteristics between patients who initiated insulin following 1 OAD, 2 OADs, or $\geq 3$ OADs. We noted that, in a real-world setting, the factors that appeared to affect the timing of insulin initiation (whether after 1 OAD, 2 OADs, or $\geq 3$ OADs) were the presence of comorbidities (macrovascular disease), cost, and risk of hypoglycemia. In other words, those who started insulin earlier (following only 1 OAD) were those with higher comorbidities and higher risk of hypoglycemia, and who incurred higher costs at baseline because of their more intensive health care utilization.

Age may also play an important role in the consideration by clinicians to initiate insulin therapy. As shown in Table 1, the age of patients in the 1 OAD group was significantly lower compared with the 2 OADs and $\geq 3$ OADs groups, although the numerical difference was not that large. Our study design, that is, including patients who had initiated a basal insulin, does not allow us to assess whether initiation of basal insulin was more common in younger or older patients. The younger age and higher comorbidity score of patients in the 1 OAD group 
could reflect that clinicians might believe that the earlier age that complications or comorbidities occur, the quicker they should initiate insulin therapy, as they might believe this group has the most to lose by delaying insulin treatment.

After initiation of basal insulin therapy, patients in all 3 groups had significant improvements in Alc levels, reductions in the proportion of patients experiencing hypoglycemia, decreases in most types of health care resource utilization, and lower health care costs compared with baseline when only OADs were used. Where evaluated, these benefits were achieved in the first 6 months after initiation of basal insulin and maintained for the second 6-month period of follow-up. Hypoglycemia forms an exception in that it increased slightly during the first 6 months of the follow-up period before reducing, compared with baseline, during the second 6 months.

Although there is substantial evidence from clinical studies regarding the benefits of early insulin therapy initiation, ${ }^{4-6,22}$ real-world outcomes data are limited. In addition, very few studies have specifically compared clinical outcomes following insulin therapy initiation after previous treatment with different numbers of OADs, ${ }^{27,28}$ and this study is the first to our knowledge to evaluate economic outcomes in this setting.

A previous pooled analysis of 11 clinical studies of insulin glargine compared clinical outcomes according to whether patients were treated with 0-1 or 2 OADs before insulin initiation. ${ }^{27}$ The results showed a significantly greater reduction in Alc from baseline to Week 24 in patients in the 0-1 OAD group compared with the 2 OADs group $(-1.8 \%$ vs. $-1.7 \% ; P=0.020)$. In addition, there was a significantly lower proportion of patients who experienced hypoglycemia in the 0-1 OAD group compared with those in the 2 OADs group (41.6\% vs. $62.9 \%$; $P<0.001){ }^{27}$

In the present study, there was a transient increase in the proportion of patients experiencing overall hypoglycemia during the first 6 months following initiation of basal insulin therapy, followed by a decrease during the second 6 months after initiation of insulin compared with those during the baseline period. This pattern may reflect increased risk of hypoglycemia as patients start using insulin (first 6 months), but a decreased risk of hypoglycemia as patients become more capable of managing their diabetes and adjusting their insulin according to their needs (second 6 months). Of note, hypoglycemia requiring hospitalization or an ED visit declined in the first and second 6 months of the analysis. These results are reassuring given that fear of hypoglycemia is one of the major barriers to initiating insulin therapy. ${ }^{18}$

Other studies have also shown that delaying initiation of insulin therapy might not achieve optimal outcomes. A retrospective database analysis of patients with T2DM in the United Kingdom found that mean Alc levels before insulin initiation increased linearly with the number of OADs prescribed
(1 OAD, 9.5\%; 2 OADs, 9.6\%; 3 OADs, 9.7\%; and 4 OADs, $10.1 \%){ }^{28}$ Patients in all of these groups had a marked deterioration in Alc levels prior to insulin initiation. ${ }^{29}$ In addition, escalating OAD therapy was shown to increase the number of years that patients were exposed to hyperglycemia and was incrementally less effective at reducing Alc levels. ${ }^{28}$

An economic modeling study indicated that early versus delayed insulin initiation provides benefits in terms of life expectancy and quality-adjusted life expectancy gained. ${ }^{29}$ In addition, by decreasing the risk of diabetes-related complications, early insulin initiation may reduce the cost burden associated with managing these complications. ${ }^{29}$ In fact, a recent publication based on a Monte Carlo microsimulation model demonstrated that earlier initiation of insulin was associated with a higher proportion of patients achieving their Alc target, decreased risk of diabetes-related complications and death, and cost savings, but an increase in hypoglycemic events. ${ }^{30}$ However, the long-term impact of insulin therapy on diabetes-related complications and associated costs remains to be determined. ${ }^{31}$

Taken together, these clinical and economic data provide a strong argument for the need for early initiation of insulin therapy in patients with T2DM.

The choice of second- and third-line therapy following initial OAD failure lacks consensus and has therefore been the subject of several large meta-analyses..$^{32,33}$ These meta-analyses comparing Alc levels, body weight, hypoglycemia, quality of life, diabetes-related complications, and other outcomes in patients receiving OADs, GLP-1 receptor agonists, or insulins found that all therapies resulted in clinically relevant decreases in Alc, but differences between compounds mainly related to risk of hypoglycemia or change in body weight. ${ }^{32,33}$

A recent real-world study analyzed outcomes between patients whose diabetes remained uncontrolled on 2 OADs and who received 1 of the 3 different third-line options as recommended by the ADA guidelines; namely, addition of a third OAD, GLP-1 receptor agonists, or insulin. This study found that physicians seemed reluctant to prescribe injectable agents for patients with uncontrolled T2DM despite multiple OAD treatment. In fact, more than $75 \%$ of patients were prescribed a third OAD, an addition that did not translate into better glycemic control and may not necessarily be a long-term cost-saving solution. ${ }^{34}$ Therefore, future studies should be conducted to provide more insights into how each of the different treatment approaches following OAD failure (second/third OAD, GLP-1 receptor agonist, or insulin) affects several short-term and longterm diabetes-related, as well as economic, outcomes.

Treatment-emergent adverse events associated with longterm antidiabetes therapy may not be identified in studies with a 1-year follow-up period. In a recent report comprising more than a median of 50 months of follow-up, for example, the 
addition of insulin by patients using metformin was associated with an increased risk of nonfatal cardiovascular events and death compared with the addition of a sulfonylurea. ${ }^{35}$ Thus, studies with longer duration may be warranted. Furthermore, given the large number of OADs available and the different mechanism of actions, an interesting research question to also pursue in the future would be to investigate the impact of the sequence and the types of OADs used in combined therapy on clinical and economic outcomes in T2DM.

In the present study, discontinuation rates with insulin treatment for 1 year ranged from $21.4 \%$ to $36.0 \%$, consistent with previous studies reporting that $71.0 \%-77.0 \%$ of patients with T2DM adhere to their insulin therapy. ${ }^{36,37}$ To help improve treatment adherence and, therefore, health outcomes, health care professionals need to ensure they have open communication with their patients and explore any reasons (such as hypoglycemia) for them not persisting with their insulin treatment. Strategies such as improving the engagement of the patient in disease management have been shown to improve insulin adherence. ${ }^{35}$

The present study shows that overall health care resource utilization and costs did not increase after initiation of basal insulin therapy in all 3 groups of patients. In fact, significant health care cost reduction was observed in all groups. However, because of the significant baseline differences, no comparisons were made on follow-up costs between these groups, and further studies and statistical analyses are warranted to clarify the potential economic benefits of timely initiation of insulin in patients with T2DM.

\section{Limitations}

This analysis aimed to descriptively examine the real-world treatment patterns and associated outcomes in patients initiating insulin after being treated with 1 OAD, 2 OADs, or $\geq 3$ OADs. Therefore, the study was limited by the fact that no statistical tests or modeling were conducted to compare outcomes between the 3 groups, and these analyses may be conducted in future studies using this dataset.

As there were significant differences in baseline characteristics and the study describes patients who initiated insulin at different time points, it is very likely that the differences in outcomes between groups observed after follow-up were influenced by these differences. Furthermore, in each of the groups, there is a possibility that patients may have discontinued their $\mathrm{OAD}(\mathrm{s})$ that were identified in the baseline period. The results from this real-world study should also be formally evaluated in randomized controlled studies to determine the comparative effectiveness of intervention with basal insulin after failure of different numbers and different types of OADs in a more rigorous setting.
The present study only included patients from a U.S. managed care setting. This criterion limits the generalizability of the findings to all patients in the United States with T2DM (from settings other than managed care [e.g., privately insured]) and patients living in countries other than the United States (e.g., Europe). Furthermore, the analyses were conducted using health care claims data, which could be potentially subject to coding errors such as under-identification of hypoglycemic events; particularly, mild occurrences. Important clinical information such as information on race and duration of diabetes was missing from the database. This meant that the number of OADs had to be used as a proxy for disease progression in the absence of data on duration of diabetes. In addition, because of the observational and exploratory nature of the study, data cannot be used to establish causality of the observed outcomes.

\section{Conclusions}

This observational descriptive study provides real-world evidence regarding clinical and economic benefits of initiating basal insulin therapy in patients with T2DM who were uncontrolled with 1 OAD, 2 OADs, or $\geq 3$ OADs. This analysis found that baseline patient demographics and disease characteristics were significantly different between the 3 groups, and that all patients experienced significant improvements in clinical and economic outcomes with the addition of insulin during the 1 year of follow-up irrespective of the number of OADs in their treatment regimens. Improvements observed in all 3 groups included reductions in Alc and lower health care resource utilization and costs, but these differences were of different magnitudes. In addition, in all 3 groups, initiation of insulin therapy was not associated with increased hypoglycemia. These results contribute to a growing body of evidence supporting the call for timely initiation of insulin therapy for patients with T2DM who are not maintaining glycemic control with OADs.

\section{Authors}

PHILIP A. LEVIN, MD, is Endocrinologist, MODEL Clinical Research, Greater Baltimore Medical Center, Baltimore, Maryland. STEVE ZHOU, PhD, is Senior Manager, Evidence-Based Medicine; JASVINDER GILL, PhD, is Senior Medical Director, Medical Affairs; and WENHUI WEI, PhD, MS, MBA, is Senior Director, EvidenceBased Medicine, Medical Affairs, Sanofi U.S., Bridgewater, New Jersey.

AUTHOR CORRESPONDENCE: Philip A. Levin, MD, MODEL Clinical Research, Greater Baltimore Medical Center, $6563 \mathrm{~N}$. Charles St., Ste. 400 N, Towson, MD 21204. Tel.: 443.524.1789; Fax: 410.828.0152; E-mail: pal3420@yahoo.com. 


\section{DISCLOSURES}

This study was funded by Sanofi U.S. Levin serves on an advisory panel for Sanofi U.S. Zhou, Gill, and Wei are employees of Sanofi U.S.

Zhou developed the study design and methodology, collected the data, provided statistical and analytic reflections of the data, and drafted the study reports. Wei codeveloped the study design, researched data, contributed to discussion, and reviewed the manuscript. Levin and Gill provided clinical insights for the study design and data interpretation, contributed to discussion, and reviewed the manuscript. All authors had full access to all the data in the study. Zhou is the guarantor of this work and, as such, takes responsibility for the integrity of the data and the accuracy of the data analysis.

\section{ACKNOWLEDGMENTS}

The authors gratefully acknowledge the statistical support provided by John Ling, MS, of Sanofi U.S. The authors received writing/editorial support in preparing this manuscript, which was funded by Sanofi U.S. and provided by Rosalie Gadiot, PhD, of Excerpta Medica, who wrote the initial draft of the manuscript.

\section{REFERENCES}

1. Cobble ME, Peters AL. Clinical practice in type 2 diabetes: After metformin and lifestyle, then what? J Fam Pract. 2009;58(11 Suppl Clinical):S7-14.

2. Shomali M. Add-on therapies to metformin for type 2 diabetes. Expert Opin Pharmacother. 2011;12(1):47-62.

3. Turner RC, Cull CA, Frighi V, Holman RR. Glycemic control with diet, sulfonylurea, metformin, or insulin in patients with type 2 diabetes mellitus: progressive requirement for multiple therapies (UKPDS 49). UK Prospective Diabetes Study (UKPDS) Group. JAMA. 1999;281(21):2005-12.

4. Holman RR, Paul SK, Bethel MA, Matthews DR, Neil HA. 10-year follow-up of intensive glucose control in type 2 diabetes. N Engl J Med. 2008;359(15):1577-89.

5. UK Prospective Diabetes Study (UKPDS) Group. Intensive blood-glucose control with sulphonylureas or insulin compared with conventional treatment and risk of complications in patients with type 2 diabetes (UKPDS 33). Lancet. 1998;352(9131):837-53.

6. Ohkubo Y, Kishikawa H, Araki E, et al. Intensive insulin therapy prevents the progression of diabetic microvascular complications in Japanese patients with non-insulin-dependent diabetes mellitus: a randomized prospective 6-year study. Diabetes Res Clin Pract. 1995;28(2):103-17.

7. Weng J, Li Y, Xu W, et al. Effect of intensive insulin therapy on betacell function and glycaemic control in patients with newly diagnosed type 2 diabetes: a multicentre randomised parallel-group trial. Lancet. 2008;371(9626):1753-60.

8. American Diabetes Association. Standards of medical care in diabetes-2011. Diabetes Care. 2011;34(Suppl 1):S11-61.

9. Korytkowski M. When oral agents fail: practical barriers to starting insulin. Int J Obes Relat Metab Disord. 2002;26(Suppl 3):S18-24.

10. Brown JB, Nichols GA, Perry A. The burden of treatment failure in type 2 diabetes. Diabetes Care. 2004;27(7):1535-40.

11. Rubino A, McQuay LJ, Gough SC, Kvasz M, Tennis P. Delayed initiation of subcutaneous insulin therapy after failure of oral glucose-lowering agents in patients with Type 2 diabetes: a population-based analysis in the UK. Diabet Med. 2007;24(12):1412-18.

12. American Diabetes Association. Standards of medical care in diabetes2012. Diabetes Care. 2012;35(Suppl 1):S11-63.
13. American Diabetes Association. Standards of medical care in diabetes2013. Diabetes Care. 2013;36(Suppl 1):S11-66.

14. American Diabetes Association. Standards of medical care in diabetes2014. Diabetes Care. 2014;37(Suppl 1):S5-13.

15. Garber AJ, Abrahamson MJ, Barzilay JI, et al.; American Association of Clinical Endocrinologists. AACE comprehensive diabetes management algorithm 2013. Endocr Pract. 2013;19(2):327-36.

16. Polonsky WH, Fisher L, Guzman S, Villa-Caballero L, Edelman SV. Psychological insulin resistance in patients with type 2 diabetes: the scope of the problem. Diabetes Care. 2005;28(10):2543-45.

17. Karter AJ, Subramanian U, Saha C, et al. Barriers to insulin initiation: the translating research into action for diabetes insulin starts project. Diabetes Care. 2010;33(4):733-35.

18. Meece J. Dispelling myths and removing barriers about insulin in type 2 diabetes. Diabetes Educ. 2006;32(1 Suppl):9S-18S.

19. Davidson MB. Early insulin therapy for type 2 diabetic patients: more cost than benefit. Diabetes Care. 2005;28(1):222-24.

20. Baser O, Wei W, Baser E, Xie L. Clinical and economic outcomes in patients with type 2 diabetes initiating insulin glargine disposable pen versus exenatide BID. J Med Econ. 2011;14(6):673-80.

21. Xie L, Wei W, Pan C, Du J, Baser O. A real-world study of patients with type 2 diabetes initiating basal insulins via disposable pens. Adv Ther 2011;28(11):1000-11.

22. Asche CV, Bode B, Busk AK, Nair SR. The economic and clinical benefits of adequate insulin initiation and intensification in people with type 2 diabetes mellitus. Diabetes Obes Metab. 2012;14(1):47-57.

23. Nathan DM, Buse JB, Davidson MB, et al. Management of hyperglycemia in type 2 diabetes: a consensus algorithm for the initiation and adjustment of therapy: a consensus statement from the American Diabetes Association and the European Association for the Study of Diabetes. Diabetes Care. 2006;29(8):1963-72.

24. Inzucchi SE, Bergenstal RM, Buse JB, et al. Management of hyperglycaemia in type 2 diabetes, 2015: a patient-centred approach. Update to a Position Statement of the American Diabetes Association and the European Association for the Study of Diabetes. Diabetologia. 2015;58(3):429-42.

25. Davis SN, Wei W, Garg S. Clinical impact of initiating insulin glargine therapy with disposable pen versus vial in patients with type 2 diabetes mellitus in a managed care setting. Endocr Pract. 2011;17(6):845-52.

26. Zhao Y, Campbell CR, Fonseca V, Shi L. Impact of hypoglycemia associated with antihyperglycemic medications on vascular risks in veterans with type 2 diabetes. Diabetes Care. 2012;35(5):1126-32.

27. Fonseca V, Gill J, Zhou R, Leahy J. An analysis of early insulin glargine added to metformin with or without sulfonylurea: impact on glycaemic control and hypoglycaemia. Diabetes Obes Metab. 2011;13(9):814-22.

28. Evans ML, Sharplin P, Owens DR, Chamberlain GH, Longman AJ, McEwan P. Insulin usage in type 2 diabetes mellitus patients in UK clinical practice: a retrospective cohort-based analysis using the THIN database. Br J Diabetes Vasc Dis. 2010;10(4):178-82.

29. Goodall G, Sarpong EM, Hayes C, Valentine WJ. The consequences of delaying insulin initiation in UK type 2 diabetes patients failing oral hyperglycaemic agents: a modelling study. BMC Endocr Disord. 2009;9:19. 
30. Smolen HJ, Murphy DR, Gahn JC, Yu X, Curtis BH. The evaluation of clinical and cost outcomes associated with earlier initiation of insulin in patients with type 2 diabetes mellitus. J Manag Care Spec Pharm. 2014;20(9):968-84. Available at: http://www.amcp.org/WorkArea/ DownloadAsset.aspx?id=18474.

31. Wallia A, Molitch ME. Insulin therapy for type 2 diabetes mellitus. JAMA. 2014;311(22):2315-25.

32. McIntosh B, Cameron C, Singh SR, et al. Second-line therapy in patients with type 2 diabetes inadequately controlled with metformin monotherapy: a systematic review and mixed-treatment comparison meta-analysis. Open Med. 2011;5(1):e35-48

33. Liu SC, Tu YK, Chien MN, Chien KL. Effect of antidiabetic agents added to metformin on glycaemic control, hypoglycaemia and weight change in patients with type 2 diabetes: a network meta-analysis. Diabetes Obes Metab. 2012;14(9):810-20.
34. Levin PA, Wei W, Zhou S, Xie L, Baser O. Outcomes and treatment patterns of adding a third agent to 2 OADs in patients with type 2 diabetes.

J Manag Care Spec Pharm. 2014;20(5):501-12. Available at: http://www.amcp. org/WorkArea/DownloadAsset.aspx?id=18026.

35. Roumie CL, Greevy RA, Grijalva CG, et al. Association between intensification of metformin treatment with insulin vs sulfonylureas and cardiovascular events and all-cause mortality among patients with diabetes. JAMA. 2014;311(22):2288-96.

36. Cramer JA, Pugh MJ. The influence of insulin use on glycemic control: How well do adults follow prescriptions for insulin? Diabetes Care. 2005;28(1):78-83.

37. Donnelly LA, Morris AD, Evans JM; DARTS/MEMO collaboration. Adherence to insulin and its association with glycaemic control in patients with type 2 diabetes. QJM. 2007;100(6):345-50. 
Health Outcomes Associated with Initiation of Basal Insulin After 1, 2, or $\geq 3$ Oral

Antidiabetes Drug(s) Among Managed Care Patients with Type 2 Diabetes

APPENDIX Median and Range of Annualized All-Cause and Diabetes-Related Health

Care Costs per Patient at Baseline and 1-Year Follow-up

\begin{tabular}{|c|c|c|c|c|c|c|}
\hline & \multicolumn{2}{|c|}{$1 \mathrm{OAD}(\mathrm{N}=19,168)$} & \multicolumn{2}{|c|}{2 OADs $(\mathrm{N}=29,112)$} & \multicolumn{2}{|c|}{$\geq 3$ OADs $(\mathrm{N}=23,708)$} \\
\hline \multicolumn{7}{|c|}{ Baseline all-cause health care costs, \$, median [min-max] } \\
\hline Total costs & 8,434 & $(2-1,200,391)$ & 7,719 & $(39-1,206,896)$ & 8,750 & $(291-1,172,542)$ \\
\hline Inpatient costs & 0 & $(0-1,099,489)$ & 0 & $(0-1,178,027)$ & 0 & $(0-1,157,067)$ \\
\hline Outpatient costs & 2,851 & $(0-668,499)$ & 2,494 & $(0-629,111)$ & 2,357 & $(0-409,104)$ \\
\hline ED costs & 0 & $(0-110,505)$ & 0 & $(0-158,211)$ & 0 & $(0-50,676)$ \\
\hline Prescription costs & 1,626 & $(2-322,603)$ & 2,712 & $(0-218,076)$ & 4,634 & $(252-170,247)$ \\
\hline \multicolumn{7}{|c|}{ Baseline diabetes-related health care costs, median [min-max] } \\
\hline Total costs & 1,772 & $(2-786,166)$ & 2,543 & $(8-575,506)$ & 4,022 & $(229-404,237)$ \\
\hline Inpatient costs & 0 & $(0-553,981)$ & 0 & $(0-568,848)$ & 0 & $(0-390,558)$ \\
\hline Outpatient costs & 744 & $(0-661,700)$ & 860 & $(0-187,381)$ & 936 & $(0-210,506)$ \\
\hline ED costs & 0 & $(0-60,742)$ & 0 & $(0-48,457)$ & 0 & $(0-26,389)$ \\
\hline Prescription costs & 125 & $(0-9,869)$ & 607 & $(0-40,821)$ & 2,355 & $(75-31,757)$ \\
\hline Supply costs & 0 & $(0-13,750)$ & 0 & $(0-7,695)$ & 34 & $(0-3,752)$ \\
\hline \multicolumn{7}{|c|}{ Follow-up all-cause health care costs, $\$$, median [min-max] } \\
\hline Total costs & 11,973 & $(85-1,700,514)$ & 10,545 & $(46-1,246,500)$ & 10,992 & $(184-1,164,173)$ \\
\hline Inpatient costs & 0 & $(0-1,656,994)$ & 0 & $(0-1,225,230)$ & 0 & $(0-1,142,018)$ \\
\hline Outpatient costs & 4,012 & $(0-861,171)$ & 3,426 & $(0-829,803)$ & 3,133 & $(0-443,292)$ \\
\hline ED costs & 0 & $(0-111,394)$ & 0 & $(0-123,174)$ & 0 & $(0-60,452)$ \\
\hline Prescription costs & 3,825 & $(30-338,347)$ & 4,559 & $(0-210,419)$ & 5,909 & $(80-178,256)$ \\
\hline \multicolumn{7}{|c|}{ Follow-up diabetes-related health care costs, \$, median [min-max] } \\
\hline Total costs & 3,927 & $(50-567,354)$ & 4,275 & $(43-543,434)$ & 5,232 & $(102-523,104)$ \\
\hline Inpatient costs & 0 & $(0-434,578)$ & 0 & $(0-538,771)$ & 0 & $(0-501,982)$ \\
\hline Outpatient costs & 970 & $(0-566,141)$ & 976 & $(0-266,125)$ & 980 & $(0-155,631)$ \\
\hline ED costs & 0 & $(0-31,974)$ & 0 & $(0-71,033)$ & 0 & $(0-32,790)$ \\
\hline Prescription costs & 1,252 & $(4-37,067)$ & 1,784 & $(0-38,787)$ & 2,993 & $(11-22,787)$ \\
\hline Supply costs & 284 & $(0-5,564)$ & 298 & $(0-8,596)$ & 339 & $(0-5,116)$ \\
\hline
\end{tabular}

